\title{
Evaluación bromatológica de la semilla de maqui (Aristotelia chilensis (Molina), Stuntz)
}

\author{
Bromatological assessment seed maqui (Aristotelia chilensis (Molina), Stuntz)
}

\author{
Ximena Araneda ${ }^{1}$, Alejandra Pacheco ${ }^{1}$, María Isabel Martínez ${ }^{1}$, Daniza Morales ${ }^{1}$
}

\begin{abstract}
RESUMEN
El objetivo de esta investigación fue evaluar las características bromatológicas de la semilla de maqui (Aristotelia chilensis). Para ello, las semillas fueron tomadas de frutos maduros colectados en la comuna de Temuco, posteriormente, los frutos fueron lavados y abiertos para retirar las semillas. Los análisis realizados fueron: proteína cruda (\%), extracto etéreo (\%), fibra cruda (\%), materia seca $(\%)$, cenizas (\%) y determinación de polifenoles. Los porcentajes encontrados en las evaluaciones fueron: 9,24\% de proteína cruda, $11,91 \%$ de extracto etéreo, $35,78 \%$ de fibra cruda, $91,97 \%$ de materia seca, $2,06 \%$ de cenizas y el contenido de polifenoles fue de $1.670 \mathrm{mg}$ equivalentes de ácido gálico por $100 \mathrm{~g}$ base materia seca. Los resultados obtenidos servirán para entregar mayores antecedentes en la toma de decisión para potenciar el uso de este producto hacia la agroindustria alimentaria.
\end{abstract}

Palabras clave: baya, polifenoles, bromatología.

\begin{abstract}
The objective of this research was to evaluate the qualitative characteristics of seed maqui (Aristotelia chilensis). To do this, the seeds were taken from ripe fruits collected in the town of Temuco, and then the fruits were washed and open to remove the seeds. The analyzes performed were: crude protein (\%), ether extract (\%), crude fiber (\%), dry matter (\%), ash (\%) and concentration of polyphenols. The percentages found in the evaluations were: $9.24 \%$ crude protein, $11.91 \%$ crude fat, $35.78 \%$ crude fiber, $91.97 \%$ dry matter, 2,06\% ash and content polyphenols was 1,670 $\mathrm{mg}$ of gallic acid equivalents per $100 \mathrm{~g}$. The results obtained will serve to provide further background in decisions making to promote the use of this product towards food agribusiness.

Key words: berry, polyphenols, bromatology.
\end{abstract}

\section{Introducción}

El maqui (Aristotelia chilensis (Molina), Stuntz) conocido también como maquei o queldrón, proviene del mapudungun cuyo significado es la baya negra producida por esta especie (Moesbach, 1992), perteneciente a la familia Elaeocarpaceae (Hoffmann et al., 1992).

Su baya tiene un alto contenido de fibra dietaria, lo que hace de este recurso un importante alimento funcional, además, constituyen una rica fuente dietética de antocianinas y antioxidantes fenólicos (Reyes-Carmona et al., 2005; Araya et al., 2006) de importancia en la prevención de enfermedades cardiovasculares, y son ricos en diversos minerales, especialmente calcio (Martínez et al., 2000; Damascos y Arribere, 2009).
A. chilensis se caracteriza por tener un elevado valor ORAC, indicador que mide la capacidad antioxidante presente en un alimento, por tanto, entre mayor es el valor ORAC, mayor es la capacidad antioxidante (Speisky, 2011). Estudios realizados por Escribano-Bailón et al. (2006) dan cuenta que su composición es particularmente rica en antocianinas donde un $34 \%$ correspondería a delfinidina-3-sambubiosido-5-glucosido. Su fruto contiene algunos alcaloides del tipo indólico y quinolínico un potencial antiaterogénico de los polifenoles de este fruto (Céspedes et al., 2008).

Según la literatura existen diversos estudios que se han realizado con esta especie, en cuanto a sus hojas y bayas; algunos autores como Avello et al. (2008), evaluaron la capacidad antioxidante de la infusión y extractos de las hojas secas, también

\footnotetext{
1 Escuela de Agronomía. Facultad de Recursos Naturales. Universidad Católica de Temuco. Rudecindo Ortega 02950. Temuco, Chile. * Autor para correspondencia: xaraneda@uct.cl
} 
Schreckinger et al. (2010), presentaron evidencias in vitro que sugieren una actividad antiinflamatoria e inhibitoria de la adipogénesis de los polifenoles del maqui. Por su parte, Misle et al. (2011), mencionan la revalorización que ha tenido el uso de colorantes comestibles, como el caso del maqui, después del descubrimiento de los efectos secundarios en los tintes sintéticos. De la misma manera, Montenegro et al. (2008) indican la gran importancia y usos potenciales para esta especie, donde reportaron el uso de la flora autóctona como fuente para la producción de miel en Chile, destacando al maqui como una de las especies que aparecen en mayor frecuencia en la fracción de polen, siendo útil como fuente de antioxidante y con potencial nutracéutico (Céspedes et al., 2010), tal como lo mencionan por Misle et al. (2011), quienes indican el uso de formulaciones en polvo para diversas aplicaciones, así como la microencapsulación de compuestos fenólicos (antocianinas).

De acuerdo a lo mencionado anteriormente, el objetivo de la presente investigación fue evaluar las características bromatológicas de la semilla de maqui (A. chilensis), para así poder entregar mayores antecedentes en la toma de decisión sobre la factibilidad de usos potenciales de este producto hacia la agroindustria alimentaria.

\section{Materiales y Métodos}

Las semillas de maqui fueron obtenidas de frutos maduros colectados en la comuna de Temuco durante la época estival. Posteriormente, estos fueron llevados al Laboratorio de Bromatología de la Escuela de Agronomía de la Universidad Católica de Temuco. Una vez allí se procedió a lavar los frutos para seguidamente partirlos y extraer las semillas contenidas dentro. Luego las semillas se molieron, con un molino (IKA A11 Basic), obteniendo una cantidad de $50 \mathrm{~g}$ peso fresco, los que se distribuyeron en tres submuestras, para luego realizarle los análisis bromatológicos correspondientes.

\section{Determinación de proteína cruda (PC)}

El método se basa en la destrucción de la materia orgánica con ácido sulfúrico concentrado, formándose sulfato de amonio, que en exceso de hidróxido de sodio, libera amoníaco, el que se destila recibiéndolo en: ácido bórico formándose borato de amonio, el que se valora con ácido sulfúrico diluido a una concentración $0,1 \mathrm{~N}$.
Se determinó utilizando el método analítico de Kjeldahl, que comprende tres etapas: digestión, destilación, y titulación. A esta determinación de proteína se le denomina cruda, porque no es una medición directa de la proteína, sino una estimación de la proteína total basada en el contenido en nitrógeno del alimento (nitrógeno x 6,25 = proteína cruda) (AOAC, 1998).

Para llevar a cabo este análisis se procedió a pesar $0,5 \mathrm{~g}$ de muestra sobre un papel tissue, en seguida se introdujo la muestra a un tubo digestor (modelo DK 20), al que se le agregó $10 \mathrm{~mL}$ de $\mathrm{H}_{2} \mathrm{SO}_{4}$ concentrado y fue sometido a ebullición durante 70 min a $400^{\circ} \mathrm{C}$, donde la muestra se digirió destruyendo la materia orgánica. Se dejó enfriar la solución y se llevó a un destilador de proteína (modelo UDK 127; Marca Velp Scientifica) donde se destiló durante 4 min, se utilizó un matraz de $500 \mathrm{~mL}$, al cual se le agregó $100 \mathrm{~mL}$ de ácido bórico y tres gotas de indicador (indicador mixto de Tashiro) y, finalmente, la solución recibida en el matraz se tituló con ácido sulfúrico diluido a una concentración $0,1 \mathrm{~N}$. El resultado se determinó a través de las siguientes fórmulas:

Nitrógeno en la muestra $(\%)=((\mathrm{A} \times \mathrm{B} \times 0,14) / \mathrm{C})$

Proteína cruda $(\%)=$ Nitrógeno en la muestra $* 6,25$

Donde: $\mathrm{A}=$ volumen del ácido sulfúrico usado en la titulación $(\mathrm{mL}), \mathrm{B}=$ concentración del ácido, $\mathrm{C}=$ peso de la muestra $(\mathrm{g})$.

\section{Determinación extracto etéreo (EE)}

Se determinó utilizando el método Soxhlet, evaluando el contenido de grasas y aceites de la muestra, usando como solvente éter de petróleo. Se pesó $1 \mathrm{~g}$ de muestra sobre un papel filtro, se envolvió y se llevó a una estufa de secado (marca Mermmet) a $105^{\circ} \mathrm{C}$ durante $24 \mathrm{~h}$, luego se sacó y dejó enfriar en un desecador durante 15 min para luego ser sometido a un extractor de grasa goldfisch (modelo 600lf, Labconco), al que se le agregó la muestra a un tubo goldfisch con éter de petróleo y se dejó en el extractor junto a un vaso precipitado donde se fue depositando la grasa, esto se realiza por un período de 4 a $5 \mathrm{~h}$. El resultado se determinó a través de la siguiente fórmula:

$$
\mathrm{EE}(\%)=100((\mathrm{~B}-\mathrm{A}) / \mathrm{C})
$$


Donde: $\mathrm{A}=$ peso del matraz limpio y seco $(\mathrm{g})$, $\mathrm{B}=$ peso del matraz con grasa $(\mathrm{g}), \mathrm{C}=$ peso de la muestra $(\mathrm{g})$.

\section{Determinación fibra cruda (FC)}

Este método, permite determinar el contenido de fibra en la muestra, después de ser digerida con soluciones de ácido sulfúrico e hidróxido de sodio y, finalmente, calcinado el residuo. Se pesó los gramos de la muestra desengrasada que fue sometida al extractor de grasas goldfisch, y se dejó enfriar en un crisol gooch para ser introducida al extractor de fibra (Marca Velp Scientifica), se le adicionó la solución de ácido sulfúrico $0,255 \mathrm{~N}$, cinco gotas de alcohol etílico y se dejó hervir por 30 min, después de realizado esto se filtró la muestra, se lavó dos veces con agua destilada hervida, posteriormente, se realizó el mismo proceso, pero con solución de $\mathrm{NaOH} 0,313 \mathrm{~N}$ y se dejó hervir durante 30 min más, luego se filtró, se lavó tres veces la muestra con agua destilada hervida, una vez con agua destilada fría y una vez con alcohol etílico. Finalizado el proceso, la muestra se llevó a una estufa a $105^{\circ} \mathrm{C}$ durante $12 \mathrm{~h}$, se dejó enfriar en el desecador y se pesó, se llevó a un horno mufla (Modelo 62700 Furnace, Marca Termolyne) a $550{ }^{\circ} \mathrm{C}$ para calcinarla, durante $8 \mathrm{~h}$, se enfrió en desecador y se pesó. Los resultados se determinaron a través de la siguiente fórmula:

$$
\mathrm{FC}(\%)=100((\mathrm{~A}-\mathrm{B}) / \mathrm{C})
$$

Donde: $\mathrm{A}=$ peso del crisol con el residuo seco (g), B = peso del crisol con la ceniza ( $\mathrm{g}), \mathrm{C}=$ peso de la muestra $(\mathrm{g})$.

\section{Determinación materia seca (MS)}

Materia seca (MS) total se denomina a la fracción del alimento que queda después que el agua ha sido eliminada. Se determinó según el método para análisis proximal según Weende descrito por Cañas (1998). Se pesó $1,0000 \mathrm{~g}$ de la muestra fresca y se llevó a estufa a $105^{\circ} \mathrm{C}$, hasta peso constante durante $24 \mathrm{~h}$, determinándose con la siguiente fórmula:

$$
\% \mathrm{M} \text { seca }=100((\mathrm{~A}-\mathrm{B}) / \mathrm{C})
$$

Donde: $\mathrm{A}=$ peso del crisol con muestra seca (g), B = peso del crisol vacío ( $\mathrm{g}$ ), C = peso de la muestra $(\mathrm{g})$.

\section{Determinación cenizas totales (CT)}

Este análisis se emplea para determinar el contenido de ceniza en los alimentos o sus ingredientes mediante la calcinación (Cañas, 1998). Se considera como el contenido de minerales totales o material inorgánico en la muestra (Nollet, 1996; AOAC, 1998). La muestra fue sometida a un proceso de incineración en un horno mufla (Modelo 62700 Furnace, Marca Thermolyne) a $550{ }^{\circ} \mathrm{C}$ durante $8 \mathrm{~h}$, posteriormente, se sacó la muestra y se dejó enfriar en el desecador. Los resultados totales, se expresan en porcentajes de cenizas en base seca, y se determinaron a través a la siguiente fórmula:

$$
\mathrm{CT}(\%)=100((\mathrm{~A}-\mathrm{B}) / \mathrm{C})
$$

Donde: $\mathrm{A}=$ peso del crisol con ceniza $(\mathrm{g}), \mathrm{B}=$ peso del crisol (g), C = peso de la muestra $(\mathrm{g})$.

\section{Determinación de polifenoles}

La extracción de los polifenoles totales se realizó de acuerdo al método de Folin Ciocalteau descrito por Singleton y Rossi (1965). Los reactivos utilizados fueron la solución acetona/agua, solución de carbonato de sodio, solución de Folin (diluida en 10 partes de agua), ácido gálico $\left(5 \mathrm{mg} \mathrm{L}^{-1} \mathrm{a}\right.$ $25 \mathrm{mg} \mathrm{L}^{-1}$ ). Se utilizaron $0,5 \mathrm{~g}$ de semilla, se preparó una curva de calibración de ácido gálico estándar, luego de 2 h se leyó la absorbancia en un espectrofotómetro (modelo $20 \mathrm{D}$, marca Milton Ray Espectronic) a una longitud de onda de $760 \mathrm{~nm}$ y los resultados se expresaron en $\mathrm{mg}$ equivalentes ácido gálico (EAG) por $100 \mathrm{~g}$ en base a materia seca.

\section{Análisis estadístico}

Los datos obtenidos en las tres submuestras analizadas se promediaron y transformaron a porcentajes, posteriormente, se compararon con la literatura encontrada.

\section{Resultados y Discusión}

A continuación se darán a conocer los resultados de las evaluaciones analizadas a la semilla de maqui expresados en base a materia seca mencionados en la Tabla 1. 
Tabla 1. Análisis químico de la semilla de A. chilensis.

\begin{tabular}{cc}
\hline Análisis & Contenido $(\%)$ \\
\hline PC & 9,24 \\
EE & 11,91 \\
FC & 35,78 \\
MS & 91,97 \\
CT & 2,06 \\
Polifenoles (mg EAG $\left.100 \mathrm{~g}^{-1}\right)$ & 1.670 \\
\hline
\end{tabular}

PC: proteína cruda, EE: extracto etéreo, FC: fibra cruda, MS: materia seca, CT: cenizas totales.

\section{Análisis proteína cruda}

En general, la cantidad de proteína presente en las semillas varía de aproximadamente $10 \%$ (en cereales) a $40 \%$ (determinadas en leguminosas y oleaginosas) de peso seco, siendo una importante fuente de proteínas en la dieta (Shewry et al., 1995). Por ejemplo, en semillas de Cucurbia sp. se han reportado valores de 58,8\% de PC (Glew et al., 2006), $22,20 \%$ y $20,82 \%$ de PC en semillas de sésamo blanco y negro, respectivamente (Margai y Sierra, 2011), y Khan et al. (2005) en bayas de Hippophae rhamnoides, reportaron valores de $19,57 \%$. En este sentido, se puede observar que los valores de PC (Tabla 1) son bajos comparados con los de otras semillas. Sin embargo, los resultados de este estudio son más altos que los reportados en semillas de frambuesa roja y negra, frambuesa, zarzamora Marion y silvestre, ya que Bushman et al. (2004) solo obtuvieron valores de 6 a $7 \%$ de proteína.

No obstante, es necesario también profundizar en el análisis de los aminoácidos presentes en estos porcentajes de proteínas a fin de evaluar la calidad de las proteínas totales, ya que, además, el contenido de proteína de la semilla depende de la ubicación de establecimiento, debido a diferencias ecológicas y condiciones climáticas de la zona y a la variedad (Aydin y Özcan, 2007).

\section{Análisis extracto etéreo}

Un aspecto a considerar en las semillas de bayas, es que estas presentan aceites especiales que han sido demandados por el mercado, al tener un perfil de ácidos grasos único con componentes interesantes (Van Hoed et al., 2009), por tanto, el componente más valioso de las semillas de bayas es el aceite (Dimić et al., 2012).
En la Tabla 1 se indica que la semilla de maqui obtuvo un $11,91 \%$ de EE, muy similar a lo reportado por Bushman et al. (2004), quienes reportaron valores de 11 a $18 \%$ de aceite en semillas de Rubus spp., y en semillas de murta Aydin y Özcan (2007) indicaron valores de $11,7 \%$, señalando que estas eran una rica fuente de ácidos grasos esenciales, siendo indicador de potencialidades nutracéuticas y utilidad industrial, por lo que sería interesante evaluar los contenidos de ácidos grasos del maqui, para ser utilizados en productos de cosmética, farmacéutica y en la industria alimentaria, ya que los ácidos grasos presentes en algunos aceites de semillas comestibles que contienen niveles significativos de otros fitoquímicos beneficiosos, tales como tocoferoles y carotenoides, por lo que los ácidos grasos presentan una alta demanda para mejorar la nutrición humana (Parry et al., 2005). Por ejemplo, Oomah et al. (2000), han reportado en semillas de frambuesa concentraciones de aceites que incluyen $\alpha-, y_{-}$, y $\delta$-tocoferol con valores de 46,$1 ; 144$ y $7,1 \mathrm{mg}$ $100 \mathrm{~g}^{-1}$ de aceite, respectivamente, y en semillas de grosella negra y fisalis, también se han reportado niveles significativos de tocoferoles (Goffman y Galleti, 2001; Ramadan y Mörsel, 2003).

Al comparar los resultados con variedades de zarzamora y frambuesa, se puede mencionar que en este estudio los resultados fueron levemente más bajo, ya que Dimić et al. (2012) reportaron valores de aceite base seca de $14,34 \%$ y $14,33 \%$ en las semillas de estas bayas, respectivamente.

\section{Análisis fibra cruda}

Se puede comparar la cantidad de fibra cruda que contiene el fruto de A. chilensis, según SchmidtHebbel et al. (1990), correspondiente a 0,8 g en $100 \mathrm{~g}$ de la parte comestible, con los resultados de la presente investigación expuestos en la Tabla 1, que muestra un valor de $35,78 \%$ de FC, lo que demuestra que la semilla contiene un alto valor de fibra, más que el fruto en sí, ya que por ejemplo, en semillas de Solanum nigrum solo se logró obtener 6,29\% de fibra cruda en base seca, valor mucho más bajo que lo reportado en este estudio (Akubugwo et al., 2007). A su vez un estudio realizado por Calvi et al. (2008) en semillas de linaza (Linum usitatissimum), demostraron un valor de $28 \%$ de $\mathrm{FC}$, lo que hace una referencia de $7,70 \%$ de diferencia, los mismos autores indican que, la semilla de linaza es una 
importante fuente nutrimental de fibra. En general, se aconseja a la población consumir comida alta en fibra ya que ofrece protección contra ciertos tipos de cáncer (Aguiar et al., 2007). Otro estudio realizado por Coelho et al. (2014), sobre semilla de chía (Salvia hispanica L.), demostraron un 23,7\% de fibra dietaria. En este sentido, la información de la semilla de maqui es desconocida y se necesita generar estudios y evaluaciones que permitan entregar nuevos antecedentes para su utilización y consumo futuro.

\section{Análisis materia seca}

De los resultados obtenidos se puede observar que el maqui presentó $91,97 \%$ de MS, mientras que Akugugwo et al. (2007), en semillas de $S$. nigrum, reportaron valores de $76,86 \%$, y en estudios realizados por Calvi et al. (2008), en semillas de linaza, presentaron un valor de $93 \%$ de MS. Además Jiménez et al. (2013) presentaron valores de semillas de rosa mosqueta (Rosa aff. rubiginosa L.), correspondientes a un 93,2\% de MS. Dichas plantas presentan similitudes del punto de su rusticidad y ambientes de desarrollo compatibles en el Sur de Chile. Sin embargo, Aydin y Özcan (2007), en semillas de murta reportaron valores mucho más bajos que en este estudio (60,3\% MS), al igual que Elagamey et al. (2013), quienes en semillas de uvas encontraron valores de $85,21 \%$ a $89,17 \%$ de MS en diferentes variedades.

\section{Análisis de cenizas totales}

Jiménez et al. (2013), reportaron $2 \mathrm{~g}$ en 100 $\mathrm{g}$ de cenizas totales. Mientras que, Mera (2008), obtuvo $3,3 \%$ en semilla de girasol y $3,41 \%$ en semillas de chía. Como se puede observar en la Tabla 1, la semilla de maqui indicó un 2,06\% de CT, similar a lo encontrado en semillas de uvas, donde Elagamey et al. (2013), obtuvieron valores de $2,52 \%$ a $2,68 \%$ de cenizas. Se ve reflejado en valores altos mencionadas primeramente, existiendo una diferencia de $1,35 \%$ con la semilla de chía, 1,24\% con semillas de girasol, no así con las semillas de mora que existe una diferencia de $0,06 \%$ de CT y $0,36 \%$ en semillas de rosa mosqueta, mientras que Khan et al. (2005), reportaron valores de 1,76 a $1,92 \%$ de cenizas en semillas de bayas de H. rhamnoides.

\section{Análisis de polifenoles}

Se puede observar (Tabla 1), que la semilla de maqui presenta $1.670 \mathrm{mg}$ EAG en $100 \mathrm{~g}$, valor alto al compararlo con semillas prensadas en frío donde se obtuvieron contenidos de polifenoles totales de $2 \mathrm{mg}$ EAG por $\mathrm{g}^{-1}$ de aceite de semillas de frambuesa roja (Parry et al., 2005). Si bien existen diferencias entre las semillas de las diversas especies vegetales, en general, se puede mencionar que los resultados de la semilla de maqui, presentan una cantidad interesante de concentración de polifenoles en consideración a que esta sería una fuente de potenciales antioxidantes, que podrían ser utilizados mediante la elaboración y procesamiento de estas semillas en algún tipo de harina y futuro aditivo a preparaciones alimentarias, ya que las semillas comestibles de algunas bayas, se caracterizan por presentar carotenoides, esteroides y compuestos fenólicos (Ramadan y Mörsel, 2003; Parry y Yu, 2004).

También se puede hacer la comparación de los polifenoles con el fruto de $A$. chilensis que según Speisky (2011), están en un rango de 1.664 a $3.116 \mathrm{mg}$ EGA $100 \mathrm{~g}^{-1}$, donde las muestras analizadas de la semilla en este estudio reportaron valores de $1.670 \mathrm{mg}$ de EGA $100 \mathrm{~g}^{-1}$, lo que demuestra un alto valor de polifenoles atribuible a la semilla.

\section{Conclusiones}

De acuerdo al estudio realizado en semillas de maqui se encontraron altos valores correspondientes a fibra cruda, extracto etéreo y polifenoles con los que se podría potenciar la agroindustria alimentaria en base a este producto.

\section{Literatura Citada}

Aguiar, A.; Rodríguez, D.; Pereira, L.; Braidotti, S.; Laguila, J.; Evelázio de Souza, N.; Visentainer, J.

2007. Effect of flaxseed oil in diet on fatty acid composition in the liver of Nile tilapia (Oreochromis niloticus). Archivos Latinoamericanos de Nutrición, 57 (3): 273-277.
Akubugwo, I.; Obasi, A.; Ginika, S.

2007. Nutritional potential of the leaves and seeds of black nightshade-Solanum nigrum L. var virginicum from Afikpo-Nigeria. Pakistan Journal of Nutrition, 6 (4): 323-326. 
AOAC.

1998. Official methods of analysis. Association of Official Analytical Chemists. Washington, D.C. 1350 p.

Araya, H.; Clavijo, C.; Herrera, C.

2006. Capacidad antioxidante de frutas y verduras cultivadas en Chile. Archivos Latinoamericanos de Nutrición, 56 (4): 361-365.

Avello, M.; Valladares, R.; Ordóñez, J.L.

2008. Capacidad antioxidante de Aristotelia chilensis (Molina) Stuntz. Revista Cubana de Plantas Medicinales, 13 (4): 1-8.

Aydin, C.; Özcan, M.M.

2007. Determination of nutritional and physical properties of myrtle (Myrtus communis L.) fruits growing wild in Turkey. Journal of Food Engineering, 79 (2): 453-458.

Bushman, B.S.; Phillips, B.; Isbel, T.; Ou, B.; Crane, J.M.;

Knapp, S.J.

2004. Chemical composition of caneberry (Rubus spp.) seeds and oils and their antioxidant potential. Journal Agricultural and Food Chemistry, 52 (26): 7982-7987.

Calvi, K.; Spreafico, F.; Teles, G.; Guzmán-Silva, M.

2008. Efecto de la semilla de linaza (Linum usitatissimum) en el crecimiento de ratas Wistar. Revista Chilena de Nutrición, 35 (4): 443-451.

Cañas, R.

1998. Alimentación y nutrición animal. Segunda edición. Pontificia Universidad Católica de Chile. Facultad de Agronomía. Santiago, Chile. 550 p.

Céspedes, C.L.; El-Hafidi, M.; Pavon, N.; Alarcón, J.

2008. Antioxidant and cardio protective activities of phenolic extracts from fruits of chilean blackberry Aristotelia chilensis (Elaeocarpaceae), maqui. Food Chemistry, 107 (2): 820-829.

Céspedes, C.L.; Valdez-Morales, M.; Ávila, J.G.; El-Hafidi, M.;

Alarcón, J.; Paredes-López, O.

2010. Phytochemical profile and the antioxidant activity of Chilean wild black-berry fruits, Aristotelia chilensis (Mol) Stuntz (Elaeocarpaceae). Food Chemistry, 119: 886-895.

Coelho, M.; de las Mercedes, M.; Salas-Mellado, M.

2014. Chemical characterization of CHIA (Salvia hispanica L.) for use in food products. Journal of Food and Nutrition Research, 2 (5): 263-269.

Damascos, M.A.; Arribere, M.

2009. Contenido mineral de hojas de maqui (Aristotelia chilensis (Molina) Stuntz) usadas en fitoterapia. Revista Cubana de Plantas Medicinales, 14 (4). Disponible en: http://www.scielo.cl/scielo.php?script=sci_arttext\&pid $=$ S0717-75182013000200010. Consultado: 20/Jun/2015.

Dimić, E.; Vujasinović, V.; Radocaj, O.; Pastor, O.

2012. Characteristics of blackberry and raspberry seeds and oils. APTEFF, 43: 1-9.

Elagamey, A.; Abdel-Wahab, M.; Shimaa, M.; Abdel-Mogib, M. 2013. Comparative study of morphological characteristics and chemical constituents for seeds of some grape table varieties. Journal of American Science, 9 (1): 447-454.

Escribano-Bailón, M.T.; Alcalde-Eon, C.; Muñoz, O.; Rivas-

Gonzalo, J.C.; Santos-Buelga, C.

2006. Anthocyanins in berries of maqui (Aristotelia chilensis (Mol.) Stuntz). Phytochemical Analysis, 17 (1): 8-14.

Glew, R.H.; Glew, R.S.; Chuang, L.; Huang, Y.; Millson, M.; Constans, D.; Vanderjagt, D.J.

2006. Amino acids, minerals and fatty acids in pumpkin seeds (Cucurbita spp.) and Cyperus esculentus nuts in the Republic of Niger. Plant Foods for Human Nutrition, 61 (2): 51-56.
Goffman, F.D.; Galletti, S.

2001. Gamma-linolenic acid and tocopherol contents in the seed oil of 47 accessions from several Ribes species. Journal of Agricultural and Food Chemistry, 49: 349-354.

Hoffmann, A.; Farga, C.; Lastra, J.; Veglozi, E. 1992. Plantas medicinales de uso común en Chile. Editorial Claudio Gay. Santiago, Chile. 273 p.

Jiménez, P.; Masson, L.; Quitral, V.

2013. Composición química de semillas de chía, linaza y rosa mosqueta y su aporte en ácidos grasos omega-3. Revista Chilena de Nutrición, 40 (2): 155-160.

Khan, Z.; Zeb, A.; Ihsanullah, I.; Qazi, I.M.

2005. Physico-chemical indices of red variety of seabuckthorn (Hippophae rhamnoides). Pakistan Journal of Food Science, 15 (1-2): 35-40.

Margai, M.; Sierra, L.

2011. The biochemical analysis of black and white sesame seeds, China. American Journal of Biochemistry and Molecular Biology, 1 (2): 145-157.

Martínez, I.; Pariago, M.; Ros, G.

2000. Significado nutricional de los compuestos fenólicos de la dieta. Archivos Latinoamericanos de Nutrición, 50 (1): 5-18.

Mera, M.

2008. Caracterización bromatológica de semillas de tres especies oleaginosas (girasol, higuerilla y chía). In: Recalde Posso, E.R.; Durán Altisent, J.M. Cultivos Energéticos Alternativos. Centro Iberoamericano de Investigación y Transferencia de Tecnología en Oleaginosas, pp. 89-98.

Misle, E.; Garrido, E.; Contardo, H.; González, W. 2011. Maqui (Aristotelia chilensis (Mol.) Stuntz) the amazing chilean tree: a review. Journal of Agricultural Science and Technology, B1: 473-482.

Moesbach, E.

1992. Botánica indígena de Chile. Andrés Bello. Santiago, Chile. 140 p.

Montenegro, G.; Gómez, M.; Díaz, J.; Pizarro, R.

2008. Application of the Chilean official standard to designate the botanical origins of honey for the characterization of the national apicultural production. Ciencia e Investigación Agraria, 35: 145-154.

Nollet, L.M.L.

1996. Handbook of food analysis. $2^{\mathrm{a}}$ edición. Marcel Dekker. New York. 330 p.

Oohmah, B.D.; Ladet, S.; Godfrey, D.V.; Liang, J.; Benoit, G. 2000. Characteristics of raspberry (Rubus idaeus L.) seed oil. Food Chemistry, 69: 187-193.

Parry, J.; Su, L.; Luther, M.; Zhou, K.; Yurawecz, M.P.; Whittaker, P.; Yu, L.

2005. Fatty acid composition and antioxidant properties of cold-pressed marionberry, boysenberry, red raspberry, and blueberry seed oils. Journal of Agricultural and Food Chemistry, 53 (3): 566-573.

Parry, J.; Yu, L.

2004. Fatty acid content and antioxidant properties of coldpressed black raspberry seed oil and meal. Journal of Food Science, 69: 189-193.

Ramadan, M.F.; Mörsel, J.T.

2003. Oil goldenberry (Physalis peruviana L.). Journal of Agricultural and Food Chemistry, 51: 969-974. 
Reyes-Carmona, J.; Youssef, G.G.; Martínez-Peniche, R.; Lila, M.A.

2005. Antioxidant capacity of fruit extracts of blackberry (Rubus sp.) produced in different climatic regions. Journal Food Science, 70: 497-503.

Schmidt-Hebbel, H.; Pennacchiotti, I.; Masson, L.; Mella, M. 1990. Tabla composición de los alimentos chilenos. $8^{\text {a }}$ edición. Facultad de Ciencias Químicas y Farmacéuticas. Departamento de Ciencias de los Alimentos y Tecnología Química. Universidad de Chile. Santiago, Chile. 62 p.

Schreckinger, M.E.; Wang, J.; Yousef, G.; Lila, M.A.; Gonzalez, E.

2010. Antioxidant capacity and in vitro inhibition of adipogenesis and inflammation by phenolic extracts of Vaccinium floribundum and Aristotelia chilensis. Journal of Agriculture Food Chemistry, 58 (16): 8966-8976.
Shewry, P.; Napier, J.; Tatham, A.

1995. Seed storage proteins: structures and biosynthesis. The Plant Cell, 7: 945-956.

Singleton, V.; Rossi, J.

1965. Colorimetry of total phenolics with phosphomoly bdicphos acid reant. American Journal Enology Viticulture, 16: 144-158. Speisky, $\mathrm{H}$.

2011. Base de datos de la actividad antioxidante (ORAC) y del contenido de polifenoles totales (PFT) en frutas producidas y/o consumidas en Chile. Instituto de Nutrición y Tecnología de los Alimentos (INTA). Disponible en http:// www.portalantioxidantes.com/documentos/BASE_ANTIOX. pdf. Consultado: 10/Ene/ 2012.

Van Hoed, V.; De Clercq, N.; Echim, C.; Andjelkovic, M.; Leber, E.; Dewettinck, K.; Verhe, R.

2009. Berry seeds: a source of specialty oils with high nutritional value. Journal of Food Lipids, 16 (1): 33-49. 
